\title{
(6) OPEN ACCESS \\ Is there a correlation between coaches' leadership styles and injuries in elite football teams? A study of 36 elite teams in 17 countries
}

\author{
Jan Ekstrand, ${ }^{1,2}$ Daniel Lundqvist, ${ }^{1,3}$ Lars Lagerbäck, ${ }^{2}$ Marc Vouillamoz, ${ }^{4}$ \\ Niki Papadimitiou, ${ }^{4}$ Jon Karlsson ${ }^{2,5}$
}

${ }^{1}$ Division of Community Medicine, Department of Medical and Health Sciences, Linkoping University, Linkoping, Sweden

${ }^{2}$ Football Research Group Linköping University, Linkoping, Sweden

${ }^{3}$ Division of Education and Sociology, Department of Behavioural Sciences and Learning, Linkoping University, Linkoping, Sweden ${ }^{4}$ UEFA, Nyon, Switzerland ${ }^{5}$ Department of Orthopaedics, Gothenburg University, Gothenburg, Sweden

Correspondence to Professor Jan Ekstrand, Health University, Hertig Karlsgatan 13BS-582 21, Linköping, Sweden;

jan.ekstrand@telia.com

Accepted 12 October 2017 Published Online First 22 October 2017
Check for updates

To cite: Ekstrand J, Lundqvist $D$, Lagerbäck $L$, et al. Br J Sports Med 2018:52:527-531.

\section{ABSTRACT \\ Background Do coaches' leadership styles affect} injury rates and the availability of players in professional football? Certain types of leadership behaviour may cause stress and have a negative impact on players' health and well-being.

Aim To investigate the transformational leadership styles of head coaches in elite men's football and to evaluate the correlation between leadership styles, injury rates and players' availability.

Methods Medical staff from 36 elite football clubs in 17 European countries produced 77 reports at four postseason meetings with a view to assessing their perception of the type of leadership exhibited by the head coaches of their respective teams using the Global Transformational Leadership scale. At the same time, they also recorded details of individual players' exposure to football and time-loss injuries.

Results There was a negative correlation between the overall level of transformational leadership and the incidence of severe injuries (rho $=-0.248 ; n=77$; $p=0.030$ ); high levels of transformational leadership were associated with smaller numbers of severe injuries. Global Transformational Leadership only explained $6 \%$ of variation in the incidence of severe injuries $\left(r^{2}=0.062\right)$. The incidence of severe injuries was lower at clubs where coaches communicated a clear and positive vision, supported staff members and gave players encouragement and recognition. Players' attendance rates at training were higher in teams where coaches gave encouragement and recognition to staff members, encouraged innovative thinking, fostered trust and cooperation and acted as role models.

Conclusions There is an association between injury rates and players' availability and the leadership style of the head coach.

\section{INTRODUCTION}

Match, training and muscle injury rates in elite men's football have remained largely unchanged overall since $2000 .^{12}$ This would suggest that preventive strategies targeting player-related risk factors are not, on their own, sufficient to significantly reduce injury rates at elite level. ${ }^{3}$ Accordingly, alternative risk factors need to be investigated in order to determine whether there is a correlation with injury rates and allow the most appropriate preventive measures to be adopted. ${ }^{3}$

When the chief medical officers of the clubs participating in the UEFA Elite Club Injury Study ${ }^{1}$ were asked for their views on the most important risk factors contributing to injuries, the four most common factors listed were: (1) the workload imposed on players, (2) players' well-being, (3) the quality of internal communication and (4) the head coach's leadership style. ${ }^{3-5}$

Leadership involves influencing others with a view to achieving a common goal and facilitating circumstances and environments that will help to reach that goal. ${ }^{67}$ There are various ways of characterising leadership, one of which is to look at it in terms of three major leadership styles: transformational (democratic/participative), transactional (authoritarian/directive) and laissez-faire. ${ }^{8}$ Transformational leadership involves motivating and inspiring followers to go beyond their self-interest for the benefit of collective interests by providing vision, meaning, challenges and stimulation. ${ }^{8}$ Transactional leadership is based on rewarding and disciplining followers on the basis of their achievements or failures, while laissez-faire leadership is, in essence, an absence of leadership. ${ }^{8}$

Research in the area of sports psychology indicates that transformational leadership on the part of coaches is associated with higher levels of motivation and performance, ${ }^{9-12}$ improved development and skill gains, ${ }^{13}$ increased well-being, ${ }^{14}$ increased satisfaction, ${ }^{10} 111516$ reduced aggression, ${ }^{17}$ increased task/team cohesion ${ }^{10}{ }^{18-20}$ and increased willingness to make personal sacrifices for the good of the team. ${ }^{19}$ However, no study has yet investigated the correlation between leadership styles and physical injuries. A coach's leadership style could cause an increase in stress levels and reduce a player's ability to cope, ${ }^{21} 22$ and we know that stress, in turn, is linked to the risk of injury. ${ }^{23-26}$

We aimed to investigate the transformational leadership of coaches of elite football teams and evaluate the correlation between their leadership styles and injury rates and the availability of players for training and matches. Our hypotheses were that (1) transformational leadership is negatively correlated with injury rates and positively correlated with the availability of players for training and matches and (2) that certain elements of transformational leadership are more important than others in this regard.

\section{MATERIAL AND METHODS}

This substudy of the ongoing Elite Club Injury Study followed 36 elite football teams from 17 European countries from 2012 to 2016. At each of the study's postseason meetings, the clubs' chief medical officers assessed the leadership styles of 
their head coaches. A total of 77 reports were produced at those meetings. Data on injuries and exposure to football were also collected from the 36 teams for each of the four seasons.

The number of reports collected per club during the 4-year study period ranged from one to four, with a median of two.

\section{Data collection}

\section{Outcome measures/dependent variables: injury data}

Data on injuries were collected in accordance with the consensus statement on injury definitions and data collection procedures in studies of football (soccer) injuries, ${ }^{27}$ and the general methodology was identical to that employed by Hägglund et al. ${ }^{28}$

An injury was defined as any physical complaint suffered by a player that resulted from a football match or a training session and led to that player being unable to participate fully in a future training session or match (ie, a time-loss injury). Players were regarded as injured until such time as the club's medical staff allowed full participation in training and made the player available for selection for matches. A severe injury was defined as an injury causing an absence from training or matches lasting more than 28 days. Baseline data on players was collected when players were first included in the study, as was players' consent to participate in the study. A member of the club's medical staff recorded individual players' exposure to football (in minutes) for all training sessions and matches. Injury and attendance reports were sent to the study group once a month. Reports were checked by the study group on receipt, with prompt feedback sent to the clubs in order to correct any missing or unclear data. Each injury was coded in accordance with a modified version of Orchard Sports Injury Classification System 2.0.9.

The risk of players being injured over a season was evaluated for each team on the basis of four criteria that have previously been found to be correlated with team performance. ${ }^{29}$

i. Injury burden: (total number of days of absence/total number of hours of exposure) $\times 1000$. This variable accounts for the incidence and severity of injuries in a season.

ii. Incidence of severe injuries: (total number of injuries causing absences of more than 28 days/total number of hours of exposure) $\times 1000$.

iii. Attendance at training: average attendance rate at training over a season, expressed as a percentage.

iv. Availability for matches: average availability for matches over a season, expressed as a percentage.

Independent variable: coaches' leadership styles

The Global Transformational Leadership (GTL) scale was used by medical staff to assess their coaches' leadership styles. The GTL scale is a reliable and appropriate tool for assessing transformational leadership. ${ }^{30}$ Team doctors were asked seven questions relating to their perception of the transformational leadership behaviours of their club's head coach. A 5-point Likert scale ranging from 'rarely or never' (1) to 'very frequently, if not always' (5) was used as a response format. Higher numbers denoted a more transformational leadership style. The seven scores were then combined to establish a mean score for each coach.

\section{Statistical analysis}

Spearman's rank correlation coefficient (rho) was used to assess the relationship between GTL scores and the four outcome measures; that is, (1) injury burden, (2) incidence of severe injuries, (3) attendance at training and (4) availability for matches. The scores for the seven GTL questions were combined to produce an overall score, and teams were placed in three groups depending on whether their coach was considered to display low (1-2), moderate (3) or high (4-5) levels of transformational leadership. Differences between the low, moderate and high groups in terms of the four outcome measures were analysed using Kruskal-Wallis (KW) tests. If the results of the KW tests were statistically significant, comparisons of individual groups (low vs moderate, low vs high and moderate vs high) were carried out using Mann-Whitney tests.

In addition, effect sizes ( $r$ ) were calculated using the results of the Mann-Whitney tests, whereby 0.1 was classified as a small effect, 0.3 was regarded as a medium-sized effect and 0.5 was classified as a large effect, as suggested by Cohen (1988). ${ }^{31}$ All analyses were two sided, and the significance level was set at $p<0.05$. Bonferroni correction was used for the comparison of individual groups, with the significance level set at $\mathrm{p}<0.0167$. IBM SPSS Statistics for Windows V.23.0 was used for all analysis.

\section{RESULTS}

There was a negative correlation between the overall GTL score and the incidence of severe injuries $(\mathrm{rho}=-0.248 ; \mathrm{n}=77$; $\mathrm{p}=0.030$ ), with high levels of transformational leadership being associated with smaller numbers of severe injuries. However, the coefficient of determination $\left(r^{2}=0.062\right)$ shows that the GTL score explained only $6 \%$ of total variation in the incidence of severe injuries. There was no significant correlation between the GTL score and the injury burden, attendance at training or availability for matches.

\section{Correlation between individual behaviours and injury rates and players' availability \\ Vision (coach communicates a clear and positive vision of the future)}

As table 1 shows, there was significant variation in the incidence of severe injuries across the high, moderate and low groups for this aspect of leadership. The incidence of severe injuries was lower for the group that was rated high in terms of visionary leadership relative to the group that was rated low $(p=0.005$; $\mathrm{r}=0.37)$.

Staff development (coach treats staff as individuals, supporting and encouraging their development)

There was significant variation in the incidence of severe injuries and attendance at training across the high, moderate and low groups for this aspect of leadership (see table 2). The high group had a lower incidence of severe injuries and higher attendance

Table 1 Vision: KW tests for injury burden, incidence of severe injuries, attendance at training and availability for matches across low, moderate and high groups

\begin{tabular}{|c|c|c|c|c|}
\hline & $\begin{array}{l}\text { Low } \\
(n=16)\end{array}$ & $\begin{array}{l}\text { Moderate } \\
(n=20)\end{array}$ & $\begin{array}{l}\text { High } \\
(n=41)\end{array}$ & \\
\hline & Median & Median & Median & $\mathrm{p}$ Value \\
\hline Injury burden* & 147.9 & 99.5 & 108.1 & 0.091 \\
\hline Incidence of severe injuriest & 1.4 & 1.0 & 1.0 & 0.020 \\
\hline Attendance at training (\%) & 81.6 & 85.2 & 84.0 & 0.143 \\
\hline Availability for matches (\%) & 86.8 & 87.7 & 87.5 & 0.261 \\
\hline
\end{tabular}

${ }^{*}$ Number of severe injuries (causing more than 28 days of absence from training and matches) per 1000 hours of exposure ( $(\Sigma$ severe injuries $/ \Sigma$ hours of exposure) $\times 1000$ ).

tNumber of days of absence per 1000 hours of exposure ( $\Sigma$ days of absence/ $\Sigma$ hours of exposure $\times 1000$ ).

KW, Kruskal-Wallis. 
Table 2 Staff development: KW tests for injury burden, incidence of severe injuries, attendance at training and availability for matches across low, moderate and high groups

\begin{tabular}{|c|c|c|c|c|}
\hline & $\begin{array}{l}\text { Low } \\
(n=16)\end{array}$ & $\begin{array}{l}\text { Moderate } \\
(\mathrm{n}=24)\end{array}$ & $\begin{array}{l}\text { High } \\
(n=37)\end{array}$ & \\
\hline & Median & Median & Median & p Value \\
\hline Injury burden* & 148.8 & 101.7 & 114.5 & 0.125 \\
\hline Incidence of severe injuriest & 1.5 & 1.0 & 0.9 & 0.008 \\
\hline Attendance at training (\%) & 81.1 & 85.3 & 82.8 & 0.048 \\
\hline Availability for matches (\%) & 86.0 & 89.2 & 87.0 & 0.114 \\
\hline
\end{tabular}

${ }^{*}$ Number of severe injuries (causing more than 28 days of absence from training and matches) per 1000 hours of exposure ( $(\Sigma$ severe injuries $/ \Sigma$ hours of exposure) $\times 1000)$.

tNumber of days of absence per 1000 hours of exposure ( $\Sigma$ days of absence/ $\Sigma$ hours of exposure $\times 1000$ )

$\mathrm{KW}$, Kruskal-Wallis

at training relative to the low group, with medium-sized effects for both variables ( 0.33 and 0.36 , respectively). There was also a difference between the low group and the moderate group, with the low group having a significantly higher incidence of severe injuries (with a median value of 1.5 per 1000 hours, compared with 1.0 for the moderate group; $\mathrm{p}=0.003 ; \mathrm{r}=0.39$ ), as well as lower attendance at training (with a median value of $81.1 \%$, compared with $85.3 \%$ for the moderate group; $\mathrm{p}=0.011$; $\mathrm{r}=0.33$ ).

Supportive leadership (coach gives encouragement and recognition to staff)

There was significant variation in the incidence of severe injuries and attendance at training across the high, moderate and low groups for this aspect of leadership (see table 3). The high group had a lower incidence of severe injuries and higher attendance at training relative to the low group, with medium-sized effects for both variables ( 0.33 and 0.36 , respectively). There was also a significant difference between the low group and the moderate group, with the low group having a higher incidence of severe injuries and lower attendance at training (with those effects totalling 0.31 and 0.28 , respectively).

Empowerment (coach fosters trust, involvement and cooperation among team members)

There was significant variation in attendance at training across the three groups for this aspect of leadership (see table 4). The

Table 3 Supportive leadership: KW tests for injury burden, incidence of severe injuries, attendance at training and availability for matches across low, moderate and high groups

\begin{tabular}{|c|c|c|c|c|}
\hline & $\begin{array}{l}\text { Low } \\
(n=20)\end{array}$ & $\begin{array}{l}\text { Moderate } \\
(n=23)\end{array}$ & $\begin{array}{l}\text { High } \\
(n=34)\end{array}$ & \multirow[b]{2}{*}{$\mathrm{p}$ Value } \\
\hline & Median & Median & Median & \\
\hline Injury burden* & 147.8 & 121.9 & 103.5 & 0.171 \\
\hline Incidence of severe injuriest & 1.3 & 1.0 & 0.7 & 0.022 \\
\hline Attendance at training (\%) & 81.2 & 84.7 & 85.2 & 0.021 \\
\hline Availability for matches (\%) & 87.1 & 86.8 & 89.2 & 0.173 \\
\hline
\end{tabular}

* Number of severe injuries (causing more than 28 days of absence from training and matches) per 1000 hours of exposure ( $(\Sigma$ severe injuries $/ \Sigma$ hours of exposure) $\times 1000)$.

†Number of days of absence per 1000 hours of exposure ( $\Sigma$ days of absence/ $\Sigma$ hours of exposure $\times 1000$ ).

KW, Kruskal-Wallis.
Table 4 Empowerment: KW tests for injury burden, incidence of severe injuries, attendance at training and availability for matches across low, moderate and high groups

\begin{tabular}{|c|c|c|c|c|}
\hline & $\begin{array}{l}\text { Low } \\
(n=17)\end{array}$ & $\begin{array}{l}\text { Moderate } \\
(n=23)\end{array}$ & $\begin{array}{l}\text { High } \\
(n=37)\end{array}$ & \\
\hline & Median & Median & Median & p Value \\
\hline Injury burden* & 149.6 & 108.1 & 112.6 & 0.169 \\
\hline Incidence of severe injuriest & 1.2 & 1.0 & 0.8 & 0.111 \\
\hline Attendance at training (\%) & 81.5 & 83.8 & 85.1 & 0.033 \\
\hline Availability for matches (\%) & 87.5 & 86.8 & 88.3 & 0.339 \\
\hline
\end{tabular}

* Number of severe injuries (causing more than 28 days of absence from training and matches) per 1000 hours of exposure ( $(\Sigma$ severe injuries/ $\Sigma$ hours of exposure) $\times 1000)$.

†Number of days of absence per 1000 hours of exposure $(\Sigma$ days of absence/ $\Sigma$ hours of exposure $\times 1000$ ).

$\mathrm{KW}$, Kruskal-Wallis

high group had a higher attendance rate than the low group (with a median value of $85.1 \%$, compared with $81.5 \%$ for the low group; $\mathrm{p}=0.011 ; \mathrm{r}=0.35)$, with a medium-sized effect.

Innovative or lateral thinking (coach encourages people to think about problems in new ways and question assumptions)

There was significant variation in attendance at training across the three groups for this aspect of leadership (see table 5). The high group had a higher attendance rate than the low group (with a median value of $85.3 \%$, compared with $81.3 \%$ for the low group; $\mathrm{p}=0.011 ; \mathrm{r}=0.32$ ), with a medium-sized effect.

Leading by example (coach is clear about his values and practises what he preaches)

There was significant variation in attendance at training and availability for matches across the three groups for this aspect of leadership (see table 6). The low group had significantly lower attendance at training than the moderate group (with a median value of $81.4 \%$, compared with $86.7 \%$ for the moderate group; $\mathrm{p}=0.006 ; \mathrm{r}=0.36$ ), as well as lower availability for matches (with a median value of $85.4 \%$, compared with $90.7 \%$ for the moderate group; $\mathrm{p}=0.027 ; \mathrm{r}=0.30)$.

Charismatic leadership (coach instils pride and respect in others and inspires players by being highly competent)

There were no significant differences between the various groups in terms of injury rates or players' availability for this

Table 5 Innovative or lateral thinking: KW tests for injury burden, incidence of severe injuries, attendance at training and availability for matches across low, moderate and high groups

\begin{tabular}{lcccccc}
\hline & $\begin{array}{l}\text { Low } \\
(\mathrm{n}=23)\end{array}$ & & $\begin{array}{l}\text { Moderate } \\
(\mathrm{n}=16)\end{array}$ & & $\begin{array}{l}\text { High } \\
(\mathrm{n}=38)\end{array}$ & \\
\cline { 2 - 3 } & Median & & Median & & Median & p Value \\
\hline Injury burden* & 147.9 & & 113.8 & & 109.5 & 0.318 \\
\hline Incidence of severe injuriest & 1.2 & & 1.1 & & 0.8 & 0.313 \\
\hline Attendance at training (\%) & 81.3 & & 85.3 & & 85.3 & 0.033 \\
Availability for matches (\%) & 86.3 & & 89.2 & & 88.5 & 0.202 \\
\hline
\end{tabular}

*Number of severe injuries (causing more than 28 days of absence from training and matches) per 1000 hours of exposure ( $(\Sigma$ severe injuries $/ \Sigma$ hours of exposure) $\times 1000$ ).

tNumber of days of absence per 1000 hours of exposure $(\Sigma$ days of absence/ $\Sigma$ hours of exposure $\times 1000$ ).

KW, Kruskal-Wallis. 
Table 6 Leading by example: KW tests for injury burden, incidence of severe injuries, attendance at training and availability for matches across low, moderate and high groups

\begin{tabular}{|c|c|c|c|c|}
\hline & $\begin{array}{l}\text { Low } \\
(n=23)\end{array}$ & $\begin{array}{l}\text { Moderate } \\
(n=16)\end{array}$ & $\begin{array}{l}\text { High } \\
(n=38)\end{array}$ & \multirow[b]{2}{*}{$\mathrm{p}$ Value } \\
\hline & Median & Median & Median & \\
\hline Injury burden* & 171.6 & 99.5 & 114.5 & 0.107 \\
\hline Incidence of severe injuriest & 1.4 & 0.9 & 1.0 & 0.094 \\
\hline Attendance at training (\%) & 81.4 & 86.7 & 82.7 & 0.008 \\
\hline Availability for matches (\%) & 85.4 & 90.7 & 86.9 & 0.049 \\
\hline
\end{tabular}

*Number of severe injuries (causing more than 28 days of absence from training and matches) per 1000 hours of exposure ( $(\Sigma$ severe injuries/ $\Sigma$ hours of exposure) $\times 1000$ ).

tNumber of days of absence per 1000 hours of exposure $(\Sigma$ days of absence/ $\Sigma$ hours of exposure $\times 1000$ ).

$\mathrm{KW}$, Kruskal-Wallis.

aspect of leadership. There were 39 observations in the high group, 20 in the moderate group and 18 in the low group.

\section{DISCUSSION}

Our study's main finding was that clubs where coaches used a transformational or democratic leadership style had a lower incidence of severe injuries. However, this correlation was weak, with GTL scores explaining just $6 \%$ of total variation in the incidence of severe injuries.

\section{Articulating a positive vision of the future reduces the risk of severe injuries}

We found that leadership behaviours that communicated a clear and positive vision of the future appeared to reduce the risk of severe injuries. This is in line with the idea that transformational leaders develop an image of the future of their organisation and communicate that vision to their subordinates. ${ }^{30}$ Identifying new opportunities for the team and providing guidance regarding the future could increase players' expectations and intrinsic motivation and result in extra effort. ${ }^{32}$

\section{Staff development is important in order to avoid severe injuries and increase attendance at training}

In our study, a leadership style that treated staff as individuals and supported and encouraged their development was associated with a $4 \%$ increase in attendance rates at training and a $33 \%$ decline in the incidence of severe injuries (comparing low levels of transformational leadership with moderate or high levels).

\section{Supportive leadership has a positive impact}

A supportive leadership style, whereby the coach gives encouragement and recognition to staff, appears to reduce the incidence of severe injuries and increase attendance rates at training sessions. In our study, a leadership style involving low levels of support was associated with a $23 \%$ increase in the incidence of severe injuries and a $4 \%$ decline in attendance at training relative to a leadership style involving moderate levels of support.

This finding is in line with research indicating that supportive behaviour by coaches is associated with collective efficacy. ${ }^{6}$ Supportive leadership is not just important for individual players; it is important for the team as a whole. ${ }^{30}$
Fostering trust and cooperation increases attendance rates at training

We found that coaches who trust their staff and support cooperation between staff members are more likely to have higher attendance rates at training.

\section{Innovative thinking could increase attendance at training}

A leadership style that encourages people to think about problems in new ways and question assumptions seems to increase attendance at training. Coaches with this leadership style encourage players to challenge inflexible thinking and patterns of behaviour in search of improvement and excellence. ${ }^{32}$ They are also willing to take risks to achieve their goals and accept that mistakes can be an opportunity to learn. ${ }^{30}$

\section{Leading by example appears to increase availability}

In this study, low levels of clarity about a coach's values were associated with a 5\% decline in players' availability for training and matches relative to moderate levels of clarity. This highlights the coach's function as a role model that players can use as a source of guidance. ${ }^{32}$

Charisma has no impact on injury rates or players' availability It has been suggested in the past that charismatic leadership should be a good predictor of a leader's effectiveness and the performance of a business unit. ${ }^{30}$ In our study, there was no correlation between charismatic leadership and injury rates or players' availability. In a recent study in the sport of handball, charismatic leadership had a negative effect on teams' performance. ${ }^{32}$ Overall, our findings suggest that, to reduce the incidence of severe injuries and increase attendance at training, coaches should establish an interpersonal environment characterised by support, respect, trust and appreciation of staff and players. $^{6}$

\section{Theoretical framework: what might underpin these associations?}

This is a prospective epidemiological study revealing significant associations; we cannot comment on causality. We can, however, speculate as to whether a coach's democratic leadership style reduces stress on players and staff. ${ }^{26}$

Psychosocial stressors could increase the risk of injury. ${ }^{23252633-35}$ The mechanism underlying a stress-induced injury is thought to be a physiological arousal that increases muscle tension and reduces motor coordination. ${ }^{26}$ The relationship between stress and injury could stem from a decline in concentration, resulting in a reduced ability to focus. ${ }^{36}$ A performance-oriented climate is linked to increases in injury risk, as this kind of environment could promote intrateam rivalry, forcing players to risk injury. ${ }^{35}$ Thus, a coach's leadership style may impact players' stress levels and injury risk as a result of the demands placed on players, the availability of resources in order to handle demands or the general atmosphere within the team. Transformational leadership, which specifically uses visionary, motivational and supportive leadership behaviours to unite the team, ${ }^{8}$ appears highly relevant when investigating the impact of psychosocial factors on injuries. Coaches may inadvertently expose players to injury risk because their leadership fails to produce an optimally functioning team.

In a recent study of psychological factors and overuse injuries in track and field athletes, Timpka et $\mathrm{al}^{37}$ concluded that a cause of overuse injuries was not the athletic load itself, but rather the load applied in situations when the athlete's body was in need of 
rest. Since coaches are responsible for footballers' load and the balance between load and rest, it would be worth carrying out a similar study looking at football.

\section{Strengths and limitations}

The strength of this study lies in its substantial dataset, which was obtained from a homogeneous group of male professional footballers. The Elite Club Injury Study is an appropriate, reliable and useful tool for evaluating injury risk and injury patterns in elite male footballers. ${ }^{28}$ We list several limitations. First of all, this study explored the opinions of medical staff regarding their head coach's leadership. Their opinions are subjective and not necessarily representative of the views of other team members. That being said, doctors at this level work with their teams on a full-time basis and see the coaches and players every day. As a result of their medical training, they are, in our opinion, in the best position to observe and evaluate how different coaches act and cooperate in terms of their leadership styles. It could be argued that elite players are less able to provide an objective evaluation of coaches' behaviour, since they often look at their situation in the group and their relationship with the coach in a subjective manner. Moreover, they usually stay at clubs for shorter periods of time, so team doctors are in a better position to provide evaluations over time.

Second, the opinions of those medical staff could be influenced by their relationships with the head coach or by the team's results during the season. It would be interesting to look at whether prolonged exposure to the unique environment found in a football club makes certain behaviours more acceptable to individuals that work within it. A club's culture could potentially affect its doctors' perception and interpretation of the various transformational leadership categories or their ability or willingness to report objectively on the head coach's leadership.

Third, the evaluation was carried out after the end of each season. There is potential for bias in the fact that doctors at clubs with high injury rates could, as a result of those injuries, take the view that their coach has a poor leadership style.

Fourth, although the collection of data on injuries and players' availability was prospective, the data on leadership styles were only collected once, so our study captures one snapshot in time. Cross-sectional studies do not capture dynamic aspects, as relationships and behaviour can change over the course of a season,

\section{What are the findings?}

- There is a correlation between a head coach's leadership style and the incidence of severe injuries and players' availability.

- Teams whose coaches who tend to employ a transformational or democratic leadership style have a lower incidence of severe injuries in their teams. The correlation between the two explains $6 \%$ of total variation in the incidence of severe injuries.

- The incidence of severe injuries was $29 \%-40 \%$ lower in teams where coaches communicated a clear and positive vision of the future, supported staff members and gave staff encouragement and recognition.

- Attendance at training was higher in teams where coaches gave encouragement and recognised staff members, encouraged innovative thinking, fostered trust and cooperation among team members and acted as role models. and the relationships that are found may not necessarily be causative. ${ }^{6}$ Also, leadership styles could change in line with results and perceived pressure on the head coach, and this kind of change has the potential to undermine players' trust and confidence.

Fifth, this study only covered elite men's football, so the findings should not be applied to other areas of football, such as amateur, youth and women's football (or, indeed, other sports). A further limitation stems from the fact that this study only investigated transformational leadership. The correlation between injuries and other leadership styles (such as transactional leadership or laissez-faire leadership) has not yet been evaluated. Although transformational or democratic leadership has been a beneficial option in many types of organisation, ${ }^{8}$ transactional or authoritarian leadership could be more appropriate in certain settings cases. Our work opens the discussion on this domain of sports injury analysis and determinants of performance.

Acknowledgements The authors wish to thank all participating clubs, including all coaches, players and medical staff. Thanks also go to biostatistician Henrik Magnusson for his assistance with the statistical analysis.

Contributors JE and DL were responsible for the conception and design of the study. JE, MV and NP were involved in data collection over the study period. JE conducted the analyses together with the biostatistician, HM. All authors contributed to interpretation of the findings. JE wrote the first draft of the paper, which was critically revised by DL, MV, NP, LL and JK. The final manuscript was approved by all authors. JE is the study guarantor.

Funding The Football Research Group was established in Linkoping, Sweden, in cooperation with Linkoping University, using grants from UEFA, the Swedish Football Association and the Swedish Research Council for Sport Science.

Competing interests None declared.

Patient consent Obtained.

Ethics approval The design of this study was approved by the UEFA Medical Committee.

Provenance and peer review Not commissioned; externally peer reviewed.

Open Access This is an Open Access article distributed in accordance with the Creative Commons Attribution Non Commercial (CC BY-NC 4.0) license, which permits others to distribute, remix, adapt, build upon this work non-commercially, and license their derivative works on different terms, provided the original work is properly cited and the use is non-commercial. See: http://creativecommons.org/ licenses/by-nc/4.0/

(C) Article author(s) (or their employer(s) unless otherwise stated in the text of the article) 2018. All rights reserved. No commercial use is permitted unless otherwise expressly granted.

\section{REFERENCES}

1 Ekstrand J, Hägglund M, Kristenson K, et al. Fewer ligament injuries but no preventive effect on muscle injuries and severe injuries: an 11-year follow-up of the UEFA Champions League injury study. Br J Sports Med 2013;47:732-7.

2 Ekstrand J, Waldén M, Hägglund M. Hamstring injuries have increased by $4 \%$ annually in men's professional football, since 2001: a 13-year longitudinal analysis of the UEFA Elite Club injury study. Br J Sports Med 2016;50:731-7.

3 Ekstrand J. Preventing injuries in professional football: thinking bigger and working together. Br J Sports Med 2016;50:709-10.

4 Ekstrand J. Keeping your top players on the pitch: the key to football medicine at a professional level. Br J Sports Med 2013:47:723-4.

5 McCall A, Dupont G, Ekstrand J. Injury prevention strategies, coach compliance and player adherence of 33 of the UEFA Elite Club Injury Study teams: a survey of teams head medical officers. Br J Sports Med 2016;50:725-30.

6 Hampson R, Jowett S. Effects of coach leadership and coach-athlete relationship on collective efficacy. Scand J Med Sci Sports 2014;24:454-60.

7 Yukl G. Leadership in organizations. Boston: Pearson, 2013.

8 Bass B, Riggio R. Transformational leadership. Mahwah: Lawrence Erlbaum, 2006.

9 Arthur CA, Woodman T, Ong CW, et al. The role of athlete narcissism in moderating the relationship between coaches' transformational leader behaviors and athlete motivation. J Sport Exerc Psychol 2011;33:3-19.

10 Price MS, Weiss MR. Relationships among coach leadership, peer leadership, and adolescent athletes' Psychosocial and team outcomes: a test of transformational leadership theory. J App/ Sport Psychol 2013;25:265-79.

11 Rowold J. Transformational and Transactional Leadership in Martial Arts. J App/ Sport Psychol 2006;18:312-25. 
12 Soane E, Butler C, Stanton E. Followers' personality, transformational leadership and performance. Sport, Business and Management: An International Journal 2015;5:65-78.

13 Vella SA, Oades LG, Crowe TP. The relationship between coach leadership, the coach-athlete relationship, team success, and the positive developmental experiences of adolescent soccer players. Physical Education \& Sport Pedagogy 2013;18:549-61.

14 Stenling A, Tafvelin S. Transformational leadership and well-being in sports: the mediating role of need satisfaction. J App/ Sport Psychol 2014;26:182-96.

15 Hoffmann MD, Loughead TM. Investigating athlete mentoring functions and their association with leadership behaviours and protégé satisfaction. Int I Sport Exerc Psychol 2016;14:85-102.

16 Kao S-F, Tsai C-Y. Transformational leadership and athlete satisfaction: the mediating role of coaching competency. J App/ Sport Psycho/ 2016;28:469-82.

17 Tucker S, Turner N, Barling J, et al. Transformational leadership and childrens' aggression in team settings: A short-term longitudinal study. Leadersh $Q$ 2010;21:389-99.

18 Callow N, Smith MJ, Hardy L, et al. Measurement of transformational leadership and its relationship with team cohesion and performance level. J App/ Sport Psychol 2009;21:395-412.

19 Cronin LD, Arthur CA, Hardy J, et al. Transformational leadership and task cohesion in sport: the mediating role of inside sacrifice. J Sport Exerc Psychol 2015;37:23-36.

20 Smith MJ, Arthur CA, Hardy J, et al. Transformational leadership and task cohesion in sport: The mediating role of intrateam communication. Psychol Sport Exerc 2013;14:249-57.

21 Fletcher D, Hanton S. Sources of organizational stress in elite sports performers. Sport Psychol 2003;17:175-95.

22 Hanton S, Fletcher D, Coughlan G. Stress in elite sport performers: a comparative study of competitive and organizational stressors. J Sports Sci 2005;23:1129-41.

23 Ivarsson A, Johnson U. Psychological factors as predictors of injuries among senior soccer players. A prospective study. J Sports Sci Med 2010;9:347-52.

24 Ivarsson A, Johnson U, Andersen MB, et al. Psychosocial factors and sport injuries: meta-analyses for prediction and prevention. Sports Med 2017;47:353-65.
25 Ivarsson A, Johnson U, Podlog L. Psychological predictors of injury occurrence: a prospective investigation of professional Swedish soccer players. J Sport Rehabil 2013;22:19-26.

26 Williams JM, Andersen MB. Psychosocial antecedents of sport injury: review and critique of the stress and injury model'. J App/ Sport Psychol 1998;10:5-25.

27 Fuller CW, Ekstrand J, Junge A, et al. Consensus statement on injury definitions and data collection procedures in studies of football (soccer) injuries. Br J Sports Med 2006;40:193-201.

28 Hägglund $M$, Waldén $M$, Bahr R, et al. Methods for epidemiological study of injuries to professional football players: developing the UEFA model. Br J Sports Med 2005;39:340-6.

29 Hägglund $M$, Waldén $M$, Magnusson $H$, et al. Injuries affect team performance negatively in professional football: an 11-year follow-up of the UEFA Champions League injury study. Br J Sports Med 2013;47:738-42.

30 Carless SA, Wearing AJ, Mann L. A short measure of transformational leadership. J Bus Psychol 2000;14:389-405.

31 Cohen J. Statistical power analysis for the behavioral sciences. Mahwah, Lawrence Erlbaum 1988.

32 Bormann KC, Schulte-Coerne P, Diebig M, et al. Athlete characteristics and team competitive performance as moderators for the relationship between coach transformational leadership and athlete performance. J Sport Exerc Psychol 2016;38:268-81.

33 Ivarsson A, Johnson $U$, Lindwall $M$, et al. Psychosocial stress as a predictor of injury in elite junior soccer: a latent growth curve analysis. J Sci Med Sport 2014;17:366-70.

34 Johnson U, Ivarsson A. Psychological predictors of sport injuries among junior soccer players. Scand J Med Sci Sports 2011;21:129-36.

35 Steffen K, Pensgaard AM, Bahr R. Self-reported psychological characteristics as risk factors for injuries in female youth football. Scand J Med Sci Sports 2009;19:442-51.

36 Thompson NJ, Morris RD. Predicting injury risk in adolescent football players: the importance of psychological variables. J Pediatr Psychol 1994;19:415-29.

37 Timpka T, Jacobsson J, Dahlström Ö, et al. The psychological factor 'self-blame' predicts overuse injury among top-level Swedish track and field athletes: a 12-month cohort study. Br J Sports Med 2015;49:1472-7. 